\title{
Uso de medicina tradicional en parturientas atendidas en el Hospital II EsSalud Tarapoto 2018
}

Obsta. Mg. Leocadia Salas Pillaca

(ORCID: 0000-0003-1479-3024)

1salas@unsm.edu.pe

Universidad Nacional de San Martín

Tarapoto - Perú

Obsta. Dr. Delgado Bardales, José Manuel

(ORCID: 0000-0001-6574-2759)

jmdelgado@unsm.edu.pe

Universidad Nacional de San Martín

Tarapoto - Perú

Scopus autor ID: 24070333700 Código Renacyt: P0050554

Obsta. Mg. Marina Victoria Huamantumba Palomino

(ORCID: 0000-0001-6335-1471)

mvhuamantumba@unsm.edu.pe Universidad Nacional de San Martín

Tarapoto - Perú

Obsta. Mg. Consuelo Dávila Torres

(ORCID: 0000-0002-5329-5135)

cdavila@unsm.edu.pe

Universidad Nacional de San Martín

Tarapoto - Perú

Obsta. Dra. Gabriela del Pilar Palomino Alvarado

(ORCID: 0000-0002-2126-2769)

gppalomino@unsm.edu.pe

Universidad Nacional de San Martín

Tarapoto - Perú

Obsta. Mg. Natividad Lupe Macedo Rodríguez

(ORCID: 0000-0003-2425-7284)

nlmacedo@unsm.edu.pe

Universidad Nacional de San Martín

Tarapoto - Perú

Obsta. Cecilia Alhuay Suarez

(ORCID: 0000-0002-1474-7506)

ccalhuay@unsm.edu.pe

Universidad Nacional de San Martín

Tarapoto - Perú

Obsta. Dra. Gloria Francisca Quijandria Oliva

(ORCID: 0000-0002-5091-6899)

gfquijandria@unsm.edu.pe

Universidad Nacional de San Martín

Tarapoto - Perú 


\section{Use of traditional medicine in parturient women attended at Hospital II EsSalud Tarapoto 2018}

\section{RESUMEN}

El estudio tuvo como objetivo determinar el uso de la medicina tradicional en parturientas atendidas en el Hospital II EsSalud Tarapoto 2018, estudio no experimental, cuantitativo, descriptivo, la población y muestra fue 300 parturientas atendidas en el Hospital II EsSalud Tarapoto. La técnica fue la encuesta y el instrumento cuestionario. Resultados, las parturientas se caracterizaron por tener como lugar de nacimiento el departamento de San Martín con 72\%, según región natural la Región Selva con $87.14 \%$ y $68.57 \%$ proceden de zona urbana, $47.43 \%$ corresponden al grupo etáreo de 30-39 años; 46\% de educación superior no universitaria y $66.57 \%$ ocupación empleada. La mayoría de parturientas usan plantas medicinales como medicina tradicional $75 \%$, principalmente en el embarazo con $38.78 \%$ seguido del posparto con $23.19 \%$. Además, la parte más utilizada de la planta es el tallo con $39.5 \%$, seguido de las hojas con $27.8 \%$, y según modo de uso predominó la infusión con 53.6\%. Las parturientas usaron principalmente como plantas medicinales la manzanilla $22 \%$, el anís $17 \%$, la malva $13 \%$ y el apio $12 \%$, y toman de 2 a más plantas. Los beneficios de las plantas medicinales según indicación y uso corresponde a analgésico $40.30 \%$ y $39 \%$ respectivamente, digestivo $25.86 \%$ y $24 \%$ respectivamente; relajante $15.59 \%$ y $16 \%$; sólo se identificó diferencia en la indicación y acción como espasmódico $9.51 \%$ y 13\% seguido de antiinflamatorio $8.75 \%$ y $5 \%$. Según complicaciones $34 \%$ presentó alguna complicación, resaltando el inicio de parto inducido con $81 \%$ y según forma de culminación del parto el 73\% termino en parto abdominal (cesárea). En conclusión, la mayoría de parturientas usan las plantas medicinales como medicina tradicional sin indicación.

Palabras clave: medicina tradicional, parturientas, plantas medicinales 


\section{ABSTRACT}

The objective of the study was to determine the use of traditional medicine in parturient women cared for at Hospital II EsSalud Tarapoto 2018, a non-experimental, quantitative, descriptive study, the population and sample was 300 parturients cared for at Hospital II EsSalud Tarapoto. The technique was the survey and the questionnaire instrument. Results, the parturients were characterized by having the department of San Martín as their place of birth with $72 \%$, according to the natural region the Selva Region with $87.14 \%$ and $68.57 \%$ come from urban areas, $47.43 \%$ correspond to the age group of 30-39 years; $46 \%$ non-university higher education and $66.57 \%$ employed occupation. The majority of women in labor use medicinal plants as traditional medicine $75 \%$, mainly in pregnancy with $38.78 \%$ followed by postpartum with $23.19 \%$. In addition, the most used part of the plant is the stem with $39.5 \%$, followed by the leaves with $27.8 \%$, and according to the mode of use, the infusion predominated with $53.6 \%$. The women in labor used mainly chamomile $22 \%$, aniseed $17 \%$, mallow $13 \%$ and celery $12 \%$ as medicinal plants, and they take from 2 to more plants. The benefits of medicinal plants according to indication and use correspond to analgesic $40.30 \%$ and $39 \%$ respectively, digestive $25.86 \%$ and $24 \%$ respectively; relaxing $15.59 \%$ and $16 \%$; Difference in indication and action was only identified as spasmodic $9.51 \%$ and $13 \%$ followed by anti-inflammatory $8.75 \%$ and $5 \%$. According to complications, $34 \%$ presented some complication, highlighting the initiation of induced labor with $81 \%$ and according to the culmination of labor, $73 \%$ ended in abdominal delivery (cesarean section). In conclusion, the majority of women in labor use medicinal plants as traditional medicine without indication.

Keywords: traditional medicine, women in labor, medicinal plants

Artículo recibido: 15 abril 2021

Aceptado para publicación: 19 abril 2021

Correspondencia: 1salas@unsm.edu.pe

Conflictos de Interés: Ninguna que declarar 


\section{INTRODUCCIÓN}

El uso de terapias alternativas por parte de usuarios del sector salud ha incrementado en los últimos 20 años, tanto por prescripciones médicas como por la tendencia a la automedicación. Dentro las terapias, están aquellas relacionadas con plantas medicinales que son particularmente frecuentes. Sin embargo, hay aún una clara brecha entre el conocimiento popular y las evidencias surgidas del proceso investigativo. La necesidad creciente de utilizar métodos terapéuticos eficaces, seguros y de bajo costo, ha inducido el incremento significativo de los estudios que pretenden validar la medicina complementaria, alternativa o tradicional (1). Las mujeres en el parto experimentan, dolor, sangrado excesivo, frio, ansiedad y en ocasiones los remedios naturales consiguen ser más suaves que los fármacos comúnmente usados. Sin embargo, muchos de estos productos naturales carecen de suficiente investigación sobre su seguridad durante el parto (2). Aproximadamente el $80 \%$ de la población de los países en desarrollo usan la medicina tradicional derivada de plantas para tratar enfermedades en humanos, sin embargo muchos profesionales médicos no recomiendan los remedios a base de hierbas para las mujeres en el parto, ya que no existe seguridad sobre las posibles interacciones que puedan ocasionar en el parto. Son pocos los estudios que se han realizado para medir los efectos de las hierbas en las mujeres embarazadas o sobre un feto en desarrollo (3). Una práctica de la medicina tradicional durante el parto, es el consumo de infusiones de plantas medicinales como método esencial para curar ciertas molestias, preparadas a base de flores, hojas, frutos, cortezas y raíces y se administran en forma de agua aromática, cocimiento, frotaciones, emplastos, muchas de este auto administradas en base a ciertos saberes (4).

Se formuló el problema ¿ Cuál es el uso de la medicina tradicional en parturientas atendidas en el Hospital II EsSalud Tarapoto 2018?. Como objetivo general: Determinar la relación entre uso de las plantas medicinales como medicina tradicional y el inicio de parto y culminación del parto en parturientas atendidas en el Hospital II EsSalud Tarapoto 2018; y como objetivos específicos: i) Identificar las características sociodemográficas de las parturientas que usan la medicina tradicional en el Hospital II EsSalud Tarapoto 2018; ii) Conocer el porcentaje de uso de la medicina tradicional en parturientas atendidas en el Hospital II EsSalud Tarapoto 2018; iii) Establecer el tipo de planta medicinal usada como medicina tradicional en parturientas atendidas en el 
Hospital II EsSalud Tarapoto 2018.; iv) Conocer los beneficios y complicaciones del uso de la medicina tradicional de parturientas atendidas en el Hospital II EsSalud Tarapoto 2018.

Entre los estudios se resalta, Macarro D, Miguelez L, Martínez E, Martínez P, Manrique J. (2014), Fitoterapia en embarazo y lactancia: ¿beneficio o riesgo? España, Resultados, el uso de plantas medicinales en el embarazo es habitual, encontrándose el jengibre y la equinácea entre las más utilizadas. En cuanto a la etapa del embarazo de mayor consumo, se identifica el primer trimestre. El mayor porcentaje de las gestantes utiliza las plantas sin indicación médica. En cuanto a la eficacia: la equinácea es eficaz para el resfriado común e infecciones vaginales, el arándano rojo para la infección urinaria, la raíz de jengibre y la ipecacuana tienen acción antiemética, la hierba de San Juan es eficaz en la depresión postparto, la valeriana para combatir el nerviosismo y el aceite de onagra para inducir el parto. De forma general las mujeres no están educadas en cuanto a la influencia que ejerce el estilo de vida en las primeras células embrionarias. El uso de productos fitoterápicos puede ser eficaz para ayudar a solucionar muchos de los problemas del embarazo, pero es necesario ver la relación riesgo-efectividad (5). Además, Macías-Peacok B, Pérez-Jackson L, Suárez-Crespo M, Fong-Domínguez C, Pupo-Perera E. (2009), Consumo de plantas medicinales por mujeres embarazadas, Santiago de Cuba. Resultados: de 300 embarazadas, las que utilizaban plantas lo hacían principalmente en el primer trimestre. Las más consumidas fueron menta japonesa (Mentha arvensis), tilo (Justicia pectoralis), verbena (Stachytarpheta jamaicensis) y anisón (Piper auritum). Predominó el consumo sin indicación médica. Conclusiones: las plantas más consumidas existen estudios acerca de sus propiedades abortivas, citotóxicas y potencial teratogénico, por lo que es necesario educar a las embarazadas para que no se auto mediquen con dichas plantas (6).

También Perlaza N. (2016), Utilización de plantas medicinales durante el parto tradicional en la comunidad de Oyacachi, Napo 2016, Ibarra Ecuador. Resultados, las parteras utilizaron plantas medicinales como: Melloco, Contra-hierba, Linaza, Culantro, Lengua de vaca, Manzanilla, Tama-Tama, Mashua, Zanahoria Blanca, Granizo y Canayuyo, con diferentes fines terapéuticos, se elaboró una guía de observación con el objetivo de promover el cuidado de las plantas medicinales utilizadas en el parto tradicional. En conclusión, el melloco, granizo y linaza fueron las plantas más utilizadas 
durante el parto tradicional, administradas en infusión (7). Asimismo, Kennedy D, Lupattelli A, Koren G, Nordeng H. (2012), Uso de hierbas medicinales en el embarazo: resultados de un estudio multinacional, Europa, América del Norte, América del Sur y Australia. Resultados, hubo 9,459 mujeres de 23 países, de ellas 28.9\% informó el uso de hierbas medicinales durante el embarazo. La mayoría de las hierbas medicinales se usaban para las dolencias de salud relacionadas con el embarazo, como el resfrío y las náuseas. El jengibre, el arándano rojo, la valeriana y la frambuesa fueron las hierbas más utilizadas en el embarazo. La tasa más alta reportada de medicamentos a base de hierbas fue en Rusia 69\%. Las mujeres de Europa del Este (51.8\%) y Australia (43.8\%) tenían el doble de probabilidades de usar una medicina herbal contra otras regiones. Las mujeres que usaban hierbas medicinales tenían característicamente a su primer hijo, no fumadoras, que usaban ácido fólico y consumían algo de alcohol durante el embarazo. Además, las mujeres que actualmente son estudiantes y las mujeres con una educación que no es un diploma de secundaria son más propensas a usar medicamentos a base de hierbas que otras mujeres. Conclusiones, el uso de la medicina herbal durante el embarazo fue alto, aunque hubo diferencias claras entre las hierbas y los usuarios de hierbas medicinales en todas las regiones. Más comúnmente, las mujeres se automedicaron con hierbas medicinales para tratar dolencias de salud relacionadas con el embarazo. Se justifica un mayor conocimiento sobre la eficacia y la seguridad de las hierbas medicinales durante el embarazo (8).

Igualmente, Lora E, Narváez L. (2010), Creencias y costumbres que poseen las mujeres de la comunidad del Chamizo sobre el embarazo parto y puerperio desde enero a junio del 2010, Ibarra- Ecuador. Resultados: en la actualidad existen aún creencias y costumbres muy arraigadas en la gente que vive en las zonas rurales y que hacen uso de ellas en el convivir diario. En lo referente al consumo de aguas aromáticas o infusiones se indica que: de 70 mujeres el $44 \%$ tomaron agua de Paraguay durante el trabajo de parto, 35\% consumió agua de panela, y 9\% consumió agua de anís, $6.7 \%$ consumió agua de ruda, 5.5\% agua de higo. Existe comparación entre el uso de plantas medicinales en la concepción del embarazo, parto y puerperio entre mujeres jóvenes y adultas mayores. Conclusión: a pesar de los avances de la tecnología, de la salud pública, de la educación que el MSP realiza a las comunidades sobre diferentes temas, aún existen diversas creencias y costumbres sobre el embarazo, parto y puerperio (9). 
Asimismo, Fong C, Macías B, (2004), Utilización de la fitoterapia en gestantes que acuden al policlínico docente comunitario "Josue País García”. Cuba. Resultados: 38\% de las gestantes consumieron preparados de plantas medicinales. Las plantas más consumidas fueron la menta, tilo o carpintero y verbena. Las gestantes emplearon además sábila, orégano, albahaca, caña santa entre otras. La mayoría de estas plantas están contraindicadas durante el embarazo por sus propiedades abortivas, fundamentalmente durante el primer trimestre, que fue el tiempo de gestación donde se encontró mayor consumo. Se evidencia la falta de información que existe tanto en la población como en el personal médico en este sentido, y la necesidad de educar a las gestantes en cuanto a la no automedicación con estas plantas. Conclusión: Las mujeres gestantes y en lactancia, utilizan hierbas para las molestias del embarazo, acelerar el parto, disminuir el frío y promover la producción de leche. (10). Por otro lado, Pulido A, Vásquez S, Villamizar G. (2012), Uso de hierbas medicinales en mujeres gestantes y en lactancia en un hospital universitario de Bogotá, Colombia. Resultados: las gestantes usaron hierbas $33.6 \%$ con un intervalo de confianza (IC) $95 \%$ de $29.2 \%$ a $38 \% .18$ hierbas fueron usadas por las gestantes. Otras hierbas utilizadas que no se encontraban en el cuestionario fueron el té de anís, llantén, limonaria, ramo bendito, sauco, cáscara de mandarina y agua con canela. El primer y tercer trimestre de la gestación es el momento en el que más se utilizan las hierbas. El modo de uso más frecuente corresponde a la infusión y la mayoría de las prácticas son aprendidas de la madre de la gestante. La hierba más utilizada fue la manzanilla (36.7\%) surtiendo el efecto esperado en un 60\% de los casos, usada para acelerar el trabajo de parto, el frío y los cólicos. El uso más frecuente de las hierbas fue para acelerar el trabajo de parto. Las gestantes indicaron prepararse durante la gestación para la lactancia usando anís estrellado e hinojo en infusión (11).

Además, Antamba B. (2016), utilización de las plantas medicinales en el postparto, Cantón el Chaco, Provincia de Napo, período diciembre 2015 - mayo 2016, Ibarra Ecuador. Resultados: las plantas medicinales más usadas fueron el Churiyuyo y la Manzanilla, las partes más utilizadas fueron el tallo y las hojas con mayor frecuencia en el postparto mediato y la acción más buscada fue antiséptico, aplicada mayormente en modo de infusión. En 100\% de los casos coincidió la acción de la planta medicinal con la indicación. Conclusión: evidencia un gran uso de plantas medicinales durante el 
postparto principalmente buscando su acción antiséptica mediante infusiones utilizando el tallo y hojas como parte frecuente de la planta (12). También, Yampis J. (2015), plantas medicinales utilizadas durante el proceso de parto y puerperio en madres de la Comunidad Nativa Awajun de Achoaga, Amazonas 2015, los resultados evidencian que las plantas medicinales utilizadas en el parto y puerperio son: el Jengibre (Ajeg), el Piripiri (Pijipig), el Toe (Baikua), el Pahniche (Kampáanak), la Huayusa (Wais), el Mata palo (Kasua), la Toronja (Yumun), el Tsemantsem '(Tsemantsem), el Algodón (Ujush), el Matico (Matiku), la Kansa (Tuyuk:). Estas plantas se utilizan entre otras cosas para el chucaque en la gestante; corrección de la presentación podálica y situación transversa del feto, asimismo para aliviar el dolor durante el trabajo de parto, mejorar la dilatación y apurar el parto; en el puerperio se utiliza para lavar las zonas íntimas, detener las hemorragias, favorecer la expulsión de la placenta y la inversión uterina, calmar el malestar puerperal y para la recuperación de la madre a manera de tónícos y vitaminas (13).

Igualmente, Solis P, Tapia L. (2015), prácticas relacionadas con el uso de plantas medicinales en el trabajo de parto y puerperio Puesto de Salud Miramar Región La Libertad abril 2015. Perú. Resultados, las mujeres usan frecuentemente las plantas medicinales durante el parto y puerperio, entre las más usadas se encuentran la albahaca, el ajo, el culantrillo, el hinojo, la ruda que se emplean en infusión vía oral para acelerar el trabajo de parto y la manzanilla en infusión para lavados vaginales durante el puerperio como antiinflamatorio. Conclusión: Las prácticas culturales de la población en torno al parto y puerperio deben servir como eje fundamental para el cuidado integral de la salud maternal (14).

Sin embargo, Condori Z, Orellana S. (2018), Influencia del uso de infusiones de plantas medicinales en el trabajo de parto en pacientes atendidas en el Centro de Salud de Chilca, en el periodo de abril - septiembre del 2017. Huancayo Perú. Resultados, encontró que existe una influencia positiva en el uso de infusiones de plantas medicinales en el trabajo de parto. Además, existe un moderado uso de las infusiones de plantas medicinales en el trabajo de parto en $61 \%$. Las pacientes encuestadas eran mayores de 30 años de edad 47\%, nivel de educación secundario $49 \%$ y multíparas $66 \%$, reconocen al orégano como la planta medicinal más utilizadas con 45,3\%, determinaron que las finalidades de tomar estas plantas medicinales son para acelerar el 
parto con un $51 \%$ y un $49 \%$ para disminuir los dolores, en un $51 \%$ de las pacientes recibieron la información del uso de las infusiones de plantas medicinales de sus familiares de forma empírica y finalmente observaron que las gestantes siente temor al momento de ejecutar sus costumbres por miedo al maltrato que ocurre de parte del personal de salud en un 51\%. En conclusión, las plantas más consumidas son: el orégano, ruda, canela, manzanilla y dentro de estas la más utilizada es el orégano; el tipo de paciente que consume estas plantas medicinales en su mayoría son mujeres mayores de 30 años de edad de un nivel secundario y multigestas (15).

Además, León C, Tello H. (1995), Uso de la infusión de hoja de algodón (Gessypium peruvianum) y su relación con la duración del trabajo del parto en gestantes atendidas en el Hospital Regional de Loreto", San Martín - Perú. Resultados obtenidos muestran que los promedios de duración del trabajo de parto en los diferentes niveles de tratamiento en las primigestas es 11 horas con 42 minutos para el grupo control y 11 horas con 38 minutos, 10 horas con 08 minutos, 10 horas con 50 minutos, 11 horas con 55 minutos para los tratamientos B, C, D y E respectivamente. En las multigestas estos promedios son de 7 horas con 43 minutos para el grupo control y 6 horas con 36 minutos, 8 horas con 34 minutos, 8 con 55 minutos y 6 horas con 47 minutos para los tratamientos B, C, D y E respectivamente. Conclusión, la utilidad clínica de la infusión de hojas de algodón fue mínima. Según los análisis estadísticos realizados, pero obteniéndose los menores tiempos en los grupos experimentales " $C$ " y " $D$ " en primigestas y "B" y "E" en multigestas con relación al grupo control. Los principios activos encontrados en la hoja de algodón son la Gosipetina, Gositrina y Rutina (16).

Saberes y tradiciones, son aquellos conocimientos y experiencias empíricos que poseen los pueblos y son transmitidos de generación en generación y que están al margen de la educación formal (17). Muchos de estos conocimientos y prácticas provienen de aquellos gestados en la etapa de consolidación del imperio Inca y otros deben haber nacido en las culturas originarias de los Andes ecuatorianos y de cultura hispana. Todas las culturas están dotadas de valores específicos, aptos para desarrollarse, siempre que se parta del principio de que la igualdad de las culturas supone la igualdad de los pueblos. Pero esta actitud debe ser sostenida no individualmente sino por movimientos organizados que respondan a las aspiraciones de la totalidad de la población indígena (18). Históricamente los pueblos indígenas han 
sido cultores de la tierra y de los recursos naturales sin afectar el medio ambiente. Entre los kichwas de Ecuador se destacan la construcción de terrazas de cultivo, el uso de la agro-ecología, el sistema de barbecho o rotación de cultivos, el conocimiento ancestral de cultivos combinados - complementarios (19).

El Parto Humano, también llamado nacimiento, es la culminación del embarazo humano hasta el periodo de salida del bebé del útero. La edad de un individuo se define por este suceso en muchas culturas. Se considera que una mujer inicia el parto con la aparición de contracciones uterinas regulares, que aumentan en intensidad y frecuencia, acompañadas de cambios fisiológicos en el cuello uterino (20). El proceso del parto natural se categoriza en tres fases: el borramiento y dilatación del cuello uterino, el descenso y nacimiento del bebé y el alumbramiento de la placenta. El parto puede verse asistido con medicamentos como anestésicos o la oxitocina, utilizada ante complicaciones de retraso grave de alumbramiento. Junto con la episiotomía (incisión quirúrgica en la zona del perineo), todo esto no debe hacerse nunca de manera rutinaria, ya que el parto más seguro es el que evoluciona espontáneamente y en el que no se interviene innecesariamente. En algunos embarazos catalogados como de riesgo elevado para la madre o el feto, el nacimiento ocurre por cesárea: la extracción del bebé a través de una incisión quirúrgica en el abdomen (21).

Tipos de Parto, según la forma de culminación del parto: i) Parto natural: En el parto natural, el bebé nace cruzando por la vagina de la madre, con la asistencia de poca o ninguna tecnología y sin la ayuda de fármacos. En la mayoría de los centros asistenciales el parto vaginal ocurre en una posición ginecológica, con la gestante en posición decúbito dorsal, es decir, acostada sobre su espalda y sus pies sostenidos a la altura de los glúteoscon el objetivo de favorecer la comodidad del personal de salud. También existe el parto en agua caliente, en el propio hogar, en hospitales o en centros privados $(22,23)$; ii) Parto vaginal instrumental: Ocasionalmente el parto vaginal debe verse asistido con instrumentos especiales, como el vacuum o el fórceps, que prensan la cabeza del feto con la finalidad de asirlo y tirar de él fuera del canal de parto. Se indica con poca frecuencia en ciertos partos difíciles; iii) Parto abdominal o cesárea: El parto abdominal o parto por cesárea es aquel en el que el bebé no sale de forma natural por la vagina, sino que es extraido mediante una operación quirúrgica, conocida como cesárea. La cesárea es un tipo de parto que consiste en practicar un corte 
en el abdomen y el útero, a través del cual se extrae al bebé. Según el tiempo de gestación: i) Parto inmaduro: entre las 20 a las 27 semanas; ii) Parto pretérmino o prematuro: Entre las 28 y las 36 semanas; iii) Parto de término: Entre las 37 y 41 semanas; iv) Parto postérmino: Después de la semana 42 en adelante. La clasificación por la forma de inicio: i) Espontáneo: cuando se desencadena el trabajo de parto en forma normal acorde con los mecanismos fisiológicos materno-gestación; ii) Inducido: cuando se utiliza alguna técnica médica como sería la maduración cervical usando Prostaglandinas PG2alfa, o Inducción con medicamentos oxitócicos (24).

Fitoterapia, la práctica de la fitoterapia es casi tan antigua como el hombre. La fitoterapia es la medicina más antigua y probada del mundo. De forma obligada los individuos y sociedades prehistóricas mantenían un fuerte contacto con la naturaleza la cual, al principio, de una forma accidental repercutía en el hombre, ya fuera por la ingesta de plantas tóxicas o venenosas, picaduras de insecto etc. La fitoterapia pertenece al ámbito de la medicina y se relaciona estrechamente con la botánica y el estudio del metabolismo secundario vegetal, es ejercido por médicos y por fitoterapeutas. La farmacéutica tiene su aproximación a la fitoterapia en la farmacognosia, que da cuenta de los constituyentes químicos de las plantas o de sus órganos o partes y de las propiedades farmacológicas de estos (25). La Fitoterapia moderna, se basa en el conocimiento de la Farmacología, y considera los aspectos farmacodinámicos y farmacocinéticos de los medicamentos basados en plantas medicinales, en estudios preclínicos y clínicos, aunque tiene su punto de origen en el conocimiento ancestral y la experiencia de prueba y error heredada de las pasadas generaciones (26). Hoy en día la tendencia en fitoterapia es recomendar o prescribir productos estandarizados, es decir, que nos aseguren cierta cantidad de principios activos que sepamos que van a ser efectivos (26).

Importancia, si bien la medicina moderna está bien desarrollada en la mayor parte del mundo, grandes sectores de la población de los países en desarrollo todavía dependen de los profesionales tradicionales, las plantas medicinales y los medicamentos herbarios para su atención primaria (27). Las muchas y diversas formas de los productos medicinales tradicionales han evolucionado frente a entornos ampliamente diferentes en lo etnológico, cultural, climático, geográfico y aun filosófico (28). Las plantas medicinales son importantes para la investigación farmacológica y el desarrollo de 
medicamentos, no solo cuando los constituyentes de plantas se usan directamente como agentes terapéuticos sino también como materiales de base para la síntesis de los medicamentos o como modelos para compuestos farmacológicamente activos.

Uso de hierbas medicinales en el parto, la Organización Mundial de la Salud (OMS) reconoce la medicina con hierbas e invita a los países, a crear políticas sobre seguridad, eficacia, calidad, acceso y uso racional de las mismas. De igual manera hay estudios que señalan la importancia de informar sobre sus propiedades, dosis, eficacia y seguridad $(29,30)$. La cultura de la gestante incluye la realización de prácticas empíricas para el cuidado de su salud, como infusiones, sahumerios, cataplasmas, baños, baños de asiento, gotas, capsulas, cremas, solas o en mezcla, dispuesto en supermercados, expendios de hierbas y tiendas naturistas (31). Las mujeres usan las hierbas medicinales por recomendación de la madre por iniciativa propia, sugerencia de familiares, de médicos, amigos o de otras personas, como chamanes, curanderos, parteras, hierbateros, y no informan de su uso al médico (32).

Plantas medicinales usadas en la atención del parto, muchos curanderos y parteras tradicionales usan plantas medicinales para estimular el postparto. Tal vez haya plantas en su zona que se usan con buenos resultados. Algunas plantas medicinales no son muy eficaces, pero tampoco son dañinas. Otras pueden ser peligrosas. Todas las plantas medicinales tienen estos problemas, la dosis es difícil de controlar. La misma planta puede ser menos o más potente dependiendo del lugar y la tierra donde haya crecido y la temporada en que la hayan cortado (33). El estómago no funciona muy bien durante el postparto. Por eso, al cuerpo le puede costar trabajo utilizar una medicina que se toma por la boca. Cada planta puede tener sus propios riesgos (34).

Manzanilla, Familia: Compuestas Nombre científico: Matricaria chamomilla L, Propiedades medicinales: Posee acción antiinflamatoria, espasmolítica, antiulcerosa, carminativa, digestiva, bactericida, fungicida, emenagoga y sedante suave, debido a los diferentes principios activos de la planta. (36). Indicaciones: Por vía interna, en tratamiento sintomaticote trastornos digestivos, tales como espasmos gastrointestinales leves, estados inflamatorios, distensión epigástrica, flatulencia y nauseas. Por vía tópica, en inflamaciones, irritaciones e infecciones de piel y mucosas, incluyendo la cavidad bucofaríngea y las encías (enjuagues), el tracto respiratorio (inhalaciones) y la zona anogenital (baños y pomadas). Se emplea como sedante nervioso en casos de histeria, 
dolores neurálgicos, neurosis abdominal, dolores menstruales (dismenorrea), amenorrea, ansiedad e insomnio. Partes usadas en la medicina tradicional: Hojas, Flores y tallo (36).

Guaba, Familia: Mimosaceae Nombre científico: Inga spectabilis. Formas de uso recomendadas popularmente: cataplasma, infusión, baño y compresas (38). Propiedades medicinales: se recomienda como antiinflamatorio, antiséptico y cicatrizante. Partes usadas en medicina tradicional: Raíces, frutos o todas las partes aéreas (14). Camote, Familia: Convolvulaceae; Nombre científico: Ipomoea batatas Características: Planta herbácea de tallo y profundas raíces ramificadas, gruesas o delgadas, que constituyen el principal objeto de su cultivo. Propiedades medicinales: se atribuyen utilidad para el tratamiento de algunas enfermedades causadas por deficiencias nutricionales, leucemias, anemia, hipertensión, diabetes, hemorragias, aumentar la leche de las mujeres. Partes usadas en la medicina tradicional: Hojas, frutos (39, 40). Canela, Familia: Lauraceae Nombre científico: Cinnamomum zeylanicum. Propiedades medicinales: El cinmaldehido es un hipotensor y espasmolítico, incrementa el flujo sanguíneo periférico, inhibe las enzimas ciclooxigenasa y lipooxigenasa del metabolismo del ácido araquidónico. Partes utilizadas: La corteza de las ramas tiernas; tallo (41, 42). Eucalipto, Familia: Myrtaceae; Nombre científico: Eucalyptus globulus Propiedades medicinales: A las hojas se atribuyen propiedades anticatarrales, antiasmáticas, descongestivas, balsámicas y expectorantes. También se considera antiinflamatoria del aparato respiratorio y digestivo, aunque resulta tóxico a dosis elevadas, pudiendo dar lugar a síntomas digestivos, respiratorios y del riñón. Otras propiedades comúnmente atribuidas incluyen la capacidad antiséptica, antibiótica, antidiabética, antivírica, antiespasmódica, antirreumática, diaforética y antitérmica. El eucalipto es uno de los recursos botánicos más empleados en el tratamiento de afecciones respiratorias. Partes utilizadas: Hojas (43).

Higo, Familia: Moraceae Nombre científico: Ficus carica L. (44, 45). Propiedades medicinales: Expectorante, laxante, antirreumáticas, hipotensor, antibiótico, anemia, dolores menstruales y espasmódico. Partes utilizadas: Hojas, Frutos. (46, 47, 48). Nogal, Familia: Juglandaceae Nombre científico: Junglans neotropica diels. Propiedades medicinales: Las hojas secas y los frutos del nogal se usan como astringente (cicatrizante), antirraquítico y es eficaz para la escrofulosis y la anemia en 
los niños. El té da magníficos resultados para curar la diabetes, tuberculosis, desalojo de toxinas, corrige desarreglos menstruales y purifica la sangre. El té se usa también para lavados de la vagina y de la uretra en caso de flores blancas y de blenorragia. Las nueces del nogal constituyen un buen alimento. Los niños que sufren de tuberculosis en los huesos (Mal de Pott) y los niños débiles deben comer algunas nueces por día al igual que los adultos. Partes utilizadas: Hojas, frutos (49). Romero, Familia: Lamiaceae, Nombre científico: Rosmarinus officinalis L. Propiedades medicinales: Antibacteriana, antiviral, antioxidante, antiparasitaria, mejorador de memoria, liberación de dopamina, mejora la actividad neuronal, antiinflamatorio, diurética, ayuda en los cólicos menstruales, anticonceptivo, prevención cardiovascular, acción en el sistema gastrointestinal. Partes utilizadas: Hojas, tallos y flores (50, 51).

Tomillo, Familia: Lamiaceae Nombre científico: Thymus vulgaris L. Propiedades medicinales: La infusión de sus hojas sirve para combatir el asma, el catarro, la gripe, la bronquitis, la gastritis, la digestión lenta, la colitis, los gases, los espasmos gastrointestinales, la inapetencia, el reumatismo, la gota y la epilepsia. Además, sirve como antiséptico, inflamatoria - analgésica, antioxidante y cicatrizante. Partes utilizadas: Hojas, tallos y flores (52). Modo de uso - formas de administración bebidas o infusión ducha vaginal mediante un baño de asiento, llenando un recipiente ancho y poco profundo con un litro de infusión o con la cantidad necesaria para que el líquido alcance la zona afectada (1). Baño para hacer un baño medicinal, usamos plantas para tratar los nervios, hongos en la piel o heridas. Cataplasma consiste en el tratamiento tópico de preparaciones vegetales en forma de pasta, espesa y húmeda, envueltas en una tela, caliente o tibia, aplicadas sobre la parte del cuerpo afectada, con efecto emoliente, calmante y antiinflamatorio. Una vez preparada y estando bien caliente, sin colarla, empapamos un paño de tejido natural, de algodón o lino, y lo aplicamos lo más caliente posible sobre la zona a tratar. En el caso de aplicación tibia se deja enfriar una vez empapado el tejido, como es el caso de inflamaciones donde hay mucho calor o cuando hay fiebre excesiva (53).

Anis, Pimpinella anisum L., popularmente llamado el anís, anís verde, matalahúva1 o matalahúga, es una hierba de la familia de las apiáceas originaria del Asia sudoccidental y la cuenca mediterránea oriental. Uso medicinal, se utiliza la semilla, es carminativo, favoreciendo la digestión, mejora el apetito, alivia los cólicos, 
incluidos los infantiles frecuentes en bebés lactantes, náuseas y flatulencias. El anís estrellado es neurotóxico por lo que no debería administrarse a bebés y está contraindicado que lo tomen las madres lactantes. El agua de anís estimula la producción de leche en las madres lactantes, puesto que pasa a la leche materna, el bebé puede beneficiarse de sus propiedades si la madre lo consume. Puede estimular el inicio de la menstruación cuando se le toma en infusión. Para la infusión hervir una taza de agua, dejar reposar una cucharadita de semilla de anís molidas, por 10 minutos, tomar 12 tazas al día. El aceite de anís ayuda a aliviar los cólicos, y espasmos estomacales. La decocción para el cólico, se prepara al hervir 1 cucharadita de semilla en 1/2 litro de leche durante 5-10 minutos. Se debe tomar caliente y removiendo continuamente para que no se quede en el fondo de la taza su esencia. Es muy valioso contra la tos fuerte y seca, con expectoración difícil, así como problemas respiratorios asociados a la gripe. Para ello, recomiendan el consumo de limonada caliente, preparada con una infusión de anís y tipo (Bistropogum mollis). Colocando en un litro de agua hirviendo 1 cucharadita de anís de pan y 1 cucharadita de tipo, luego cernir y adicionar el jugo de 3-5 limones (Citrus $\times$ limón). Preparación que se toma 3 veces al día durante 6 a 8 días (54).

Poro, Allium ampeloprasum var. porrum, Esta planta herbácea, conocido como puerros en otros países, no solo tiene un alto valor nutritivo, sino que sus activos lo convierten en un poderoso antioxidante que revitaliza nuestro organismo contra el envejecimiento y se le considera una verdadera escoba intestinal, actuando como suave laxante además de ser protector del hígado y las funciones biliares. Propiedades, en el parto en segunda cocción, bebida, es útil para corregir el vientre duro. Esta misma preparación con vino produce el efecto contrario (estreñimiento). Conviene a los anémicos por su riqueza en hierro (1 mg.1100 g.) y magnesio (18 mg./100 g.), a los intelectuales por su riqueza en fósforo (50 rng.), bien equilibrado además con el calcio (60 mg.), y a los bronquíticos por su riqueza en azufre $(72 \mathrm{mg}$.). Sus antioxidantes favorecen a la revitalización del organismo, contrarresta el envejecimiento proporcionándonos calidad de vida en el otoño de nuestras vidas (53). Apio, (Apium graveolens) es una especie vegetal perteneciente a la familia de las Apiáceas, antiguamente conocidas como umbelíferas. Propiedades y uso, el apio nabo se utiliza en cocina. La raíz del apio nabo se puede utilizar cruda o cocida. La superficie exterior suele pelarse antes de su uso por ser demasiado dura. La carne del apio nabo es dura y compacta y de color 
hueso. El apio nabo tiene un suave sabor a apio, y a menudo se utiliza como aromatizante en sopas y guisos, aunque cada vez se utiliza más por sí solo, por lo general en puré, o en guisos, gratinados y platos al horno. En el embarazo y parto tiene efecto diurético, las semillas provocan una mayor diuresis, siendo el cocimiento de éstas, útil en caso de hipertensión arterial, afección cardíaca congestiva, ansiedad e insomnio. Así mismo, la infusión de las semillas de apio resulta beneficiosa para combatir el síndrome premenstrual que produce incomodidades poco antes de la menstruación debido a la acumulación de líquido en los tejidos (55). Algodón, las especies del género Gossypium, cultivadas para producir algodón, son plantas herbáceas y arbustos de la familia Malvaceae con unos 60 taxones específicos e infra-específicos aceptados, de los casi 380 descritos, todos oriundos de las regiones tropicales y subtropicales tanto del viejo mundo como del nuevo y unas pocas han sido introducidas en muchos otros sitios. Propiedades y usos medicinales: i) Para los dolores de cabeza o cefaleas se trituran las semillas y se forma una pasta que se aplica sobre la zona adolorida; ii) Para las fiebres y antidiarreico, preparar una infusión de las hojas, tomar en caliente una taza 2 veces al día; iii) Como un estimulante del parto para mujeres embarazadas, beber un infusión de las hojas ayuda a dilatar el útero de una parturienta; iv) Como diurético, cocer 5 gramos de raíz en agua y tomar una taza 2 veces al día; así mismo brinda beneficios en problemas dermatológicos y ginecológicos (56). Sacha orégano, Origanum vulgare, el orégano, es una planta de la familia Lamiáceas antes llamada Labiadas. Se usa como condimento. La parte utilizada son las brácteas de la inflorescencia, tanto frescas como secas, aunque secas poseen mucho más sabor y aroma. Descripción, la planta forma un pequeño arbusto achaparrado (grueso y bajito) de unos $45 \mathrm{~cm}$ de alto. Propiedades medicinales, sus propiedades han sido ampliamente estudiadas, siendo las más importantes su actividad antioxidante, antimicrobiana y, en estudios bastante primarios, antitumoral, antiséptica y también se la considera tónica y digestiva. En la medicina tradicional austriaca se ha utilizado internamente (como el té) o externamente (como pomada) para el tratamiento de trastornos del tracto gastrointestinal, tracto respiratorio y el sistema nervioso. Principios activos, el flavonol retusin se puede encontrar en Origanum vulgare, el orégano también se prepara como cigarrillos y se fuma para estimular la memoria por ser una droga nootrópica (57). Puspino, Cajanus cajan, 
el guandú, gandul, guandul, frijol de palo, frijol chícharo, palo de gandules o quinchoncho es una leguminosa arbustiva de hojas alternadas trifolioladas. Se discute sobre si su origen es África o la India, pero se cultiva hace por lo menos 3 mil años. Usos, sus semillas son utilizadas en la alimentación humana y como forraje para la alimentación animal. Contienen entre 10 y 17\% de proteína. En la medicina tradicional sus hojas se cuecen para baños que alivian la gripe y en el parto se utiliza para acelerar el trabajo de parto (58). Malva, es un género de unas 30 especies aceptadas, de las más de 350 descritas y con un número elevado (235) de taxones específicos todavía sin resolver, de plantas herbáceas en la familia Malvaceae. Se utiliza en la medicina popular por sus propiedades de limpieza de colon y como suplemento para perder peso, en el parto se utiliza como antinflamatorio y calmante del dolor (59).

\section{ESTRATEGIAS METODOLOGICAS O MATERIALES Y MÉTODOS}

El estudio fue no experimental, cuantitativo, descriptivo, de corte transversal, correlacional, y se representa en el siguiente esquema. El universo Estuvo conformado por todas las mujeres que asisten al Hospital II EsSalud Tarapoto atendidas en el año 2018. La población estuvo constituida por las parturientas atendidas en el Hospital II EsSalud Tarapoto en el año 2018, que asciende aproximadamente a 300 parturientas según dato de la oficina de estadística del Hospital II EsSalud Tarapoto. La muestra estuvo conformada por el 100\% de la población de parturientas atendidas en el Hospital II EsSalud Tarapoto año 2018, que asciende aproximadamente a 300. Como criterios de inclusión se consideró: Parturientas atendidas en el Hospital II EsSalud Tarapoto en el año 2018; parturientas que aceptan voluntariamente participar del estudio; y parturientas con condiciones físicas y mentales para responder al cuestionario. Como criterios de exclusión: mujeres que no son parturientas atendidas en el Hospital II EsSalud Tarapoto en el año 2018; Parturientas que rechazan participar del estudio y parturientas con complicaciones que le impiden participar del estudio. La técnica utilizada fue la encuesta y la revisión documentaria, la primera aplicada a las parturientas y la segunda para determinar el tipo de parto. Los instrumentos a utilizar son el cuestionario para indagar sobre el uso de la medicina tradicional y la ficha de recolección de datos adjunta para determinar los tipos de parto de la historia clínica. La encuesta constó de tres partes, la primera parte relacionada a los datos sociodemográficos, la segunda parte 
relacionada a la medicina tradicional y la tercera parte relaciona con los tipos de parto. Para el procesamiento y análisis de dato, se diseñó una base de datos en Excel 2011 y SPSS 21, que permitió la elaboración de tablas simples, de doble entrada y gráficos. Se utilizó la estadística descriptiva como: frecuencia y porcentaje.

\section{RESULTADOS Y DISCUSIÓN}

\subsection{Resultados}

Tabla 1. Características sociodemográficas - procedencia de las parturientas que usan la medicina tradicional en el Hospital II EsSalud Tarapoto 2018.

\begin{tabular}{lcc}
\hline \multicolumn{1}{c}{$\begin{array}{c}\text { Características } \\
\text { Lugar de } \\
\text { nacimiento }\end{array}$} & Fi & \% \\
\hline San Martin & $\mathbf{2 5 2}$ & $\mathbf{7 2}$ \\
Lima & 7 & 2 \\
Ucayali & 9 & 3 \\
Cajamarca & $\mathbf{2 2}$ & $\mathbf{6}$ \\
Ancash & 2 & 1 \\
Amazonas & 9 & 3 \\
Apurímac & 2 & 1 \\
Loreto & $\mathbf{3 5}$ & $\mathbf{1 0}$ \\
Huánuco & 2 & 1 \\
La Libertad & 1 & 0 \\
Lambayeque & 9 & 3 \\
Región Natural & $\mathbf{N}^{\circ}$ & $\mathbf{\%}$ \\
Costa & 18 & 5.14 \\
Sierra Sur & 2 & 0.57 \\
Sierra Central & 4 & 1.14 \\
Sierra Norte & 21 & 6.00 \\
Selva & 305 & 87.14 \\
Procedencia & $\mathbf{N}^{\circ}$ & $\mathbf{\%}$ \\
Zona Urbana & 240 & 68.57 \\
Zona Rural & 108 & 30.86 \\
Zona Urbana & & \\
Marginal & 2 & 0.57 \\
Total & $\mathbf{3 5 0}$ & $\mathbf{1 0 0 . 0 0}$ \\
\hline
\end{tabular}

Fuente: Datos propios de la investigación 
Tabla 2. Características sociodemográficas según edad, grado de instrucción y ocupación de las parturientas que usan la medicina tradicional en el Hospital II EsSalud Tarapoto 2018.

\begin{tabular}{lrr}
\hline \multicolumn{1}{c}{ Edad } & $\mathbf{N}^{\circ}$ & \% \\
\hline$<19$ años & 19 & 5.43 \\
$\mathbf{2 0}-\mathbf{2 9}$ años & $\mathbf{1 4 7}$ & $\mathbf{4 2 . 0 0}$ \\
$\mathbf{3 0}-\mathbf{3 9}$ años & $\mathbf{1 6 6}$ & $\mathbf{4 7 . 4 3}$ \\
$40-49$ años & 18 & 5.14 \\
De 50 a más años & 0 & 0.00 \\
\multicolumn{1}{c}{ Total } & $\mathbf{3 5 0}$ & $\mathbf{1 0 0 . 0 0}$ \\
\hline \multicolumn{1}{c}{ Grado de instrucción } & $\mathbf{N}^{\circ}$ & \% \\
\hline Iletrado & 0 & 0.00 \\
Primaria completa & 7 & 2.00 \\
Primaria incompleta & 2 & 0.57 \\
Secundaria completa & 60 & 17.14 \\
Secundaria incompleta & 30 & 8.57 \\
Superior universitaria & $\mathbf{9 0}$ & $\mathbf{2 5 . 7 1}$ \\
Superior no & & \\
universitario & $\mathbf{1 6 1}$ & $\mathbf{4 6 . 0 0}$ \\
\multicolumn{1}{c}{ Total } & $\mathbf{3 5 0}$ & $\mathbf{1 0 0 . 0 0}$ \\
\hline \multicolumn{1}{c}{ Ocupación } & $\mathbf{N}^{\circ}$ & \% \\
\hline Ama de casa & $\mathbf{9 8}$ & $\mathbf{2 8 . 0 0}$ \\
Agricultora & 0 & 0.00 \\
Empleada & $\mathbf{2 3 3}$ & $\mathbf{6 6 . 5 7}$ \\
Estudiante & 11 & 3.14 \\
Independiente & 8 & 2.29 \\
\multicolumn{1}{c}{ Total } & $\mathbf{3 5 0}$ & $\mathbf{1 0 0 . 0 0}$ \\
\hline \multicolumn{1}{c}{ Fuente Datos } & &
\end{tabular}

Fuente: Datos propios de la investigación

Tabla 3. Porcentaje de uso de la medicina tradicional en parturientas atendidas en el Hospital II EsSalud Tarapoto 2018.

\begin{tabular}{lccc}
\hline & Uso de plantas & $\mathbf{N}^{\circ}$ & \% \\
\hline $\mathrm{Si}$ & 263 & 75 \\
$\mathrm{No}$ & 87 & 25 \\
\multicolumn{2}{c}{$\quad$ Total } & $\mathbf{3 5 0}$ & $\mathbf{1 0 0}$ \\
\hline \multicolumn{2}{c}{ Uso según etapa } & $\mathbf{N}^{\circ}$ & $\mathbf{\%}$ \\
\hline Embarazo & 102 & 38.78 \\
Trabajo de parto & 57 & 21.67 \\
Intraparto & 43 & 16.35 \\
Posparto & 61 & 23.19
\end{tabular}




\begin{tabular}{lcc}
$\begin{array}{l}\text { Parte de la planta } \\
\text { utilizada }\end{array}$ & $\mathbf{N}^{\circ}$ & $\mathbf{\%}$ \\
\hline Tallo & 104 & 39.5 \\
Hojas & 73 & 27.8 \\
Raíz & 9 & 3.4 \\
Flores & 39 & 14.8 \\
Fruto & 27 & 10.3 \\
Semilla & 11 & 4.2 \\
Modo de uso & $\mathbf{N}^{\circ}$ & $\mathbf{\%}$ \\
\hline Infusión & 141 & 53.6 \\
Baño maría & 37 & 14.1 \\
Cocido & 85 & 32.3 \\
Total & $\mathbf{2 6 3}$ & $\mathbf{1 0 0 . 0}$ \\
\hline
\end{tabular}

Fuentes: Datos propios de la investigación

Tabla 4. Tipo de planta medicinal usada como medicina tradicional en parturientas atendidas en el Hospital II EsSalud Tarapoto 2018

\begin{tabular}{lcc}
\hline \multicolumn{1}{c}{$\begin{array}{c}\text { Plantas } \\
\text { medicinales }\end{array}$} & $\mathbf{N}^{\circ}$ & \% \\
\hline Manzanilla & 197 & 22 \\
Anís & 151 & 17 \\
Malva & 118 & 13 \\
Apio & 104 & 12 \\
Puspino & 97 & 11 \\
Poro & 75 & 8 \\
Canela & 71 & 8 \\
Sacha orégano & 54 & 6 \\
Algodón & 21 & 2 \\
Eucalipto & 6 & 1 \\
Romero & 5 & 1 \\
Higo & 1 & 0 \\
\multicolumn{1}{c}{ Total } & $\mathbf{9 0 0}$ & $\mathbf{1 0 0}$ \\
\hline \multicolumn{1}{r}{ Futes: Datos } \\
\hline
\end{tabular}

Fuentes: Datos propios de la investigación

Tabla 5. Beneficios indicación - acción del uso de las plantas medicinales como medicina tradicional de parturientas atendidas en el Hospital II EsSalud Tarapoto 2018.

\begin{tabular}{lrr}
\hline \multicolumn{1}{c}{ Indicación } & $\mathbf{N}$ & $\mathbf{\%}$ \\
\hline Analgésico & 106 & 40.30 \\
Digestivo & 68 & 25.86 \\
Relajante & 41 & 15.59 \\
Antiinflamatorio & 23 & 8.75 \\
Espasmódico & 25 & 9.51 \\
\multicolumn{1}{c}{ Total } & $\mathbf{2 6 3}$ & $\mathbf{1 0 0}$ \\
\hline
\end{tabular}




\begin{tabular}{lrr}
\hline \multicolumn{1}{c}{ Acción } & N & \% \\
\hline Analgésico & 102 & 39 \\
Espasmódico & 34 & 13 \\
Antiinflamatorio & 13 & 5 \\
Hemostático & 9 & 3 \\
Digestivo & 63 & 24 \\
Relajante & 42 & 16 \\
\multicolumn{1}{c}{ Total } & $\mathbf{2 6 3}$ & $\mathbf{1 0 0}$ \\
\hline
\end{tabular}

Fuente: Datos propios de la investigación

Tabla 6: Frecuencia de complicaciones del uso de las plantas medicinales como medicina tradicional de parturientas atendidas en el Hospital II EsSalud Tarapoto 2018.

\begin{tabular}{|c|c|c|}
\hline Complicaciones & $\mathbf{N}^{\circ}$ & $\%$ \\
\hline $\mathrm{Si}$ & 89 & 34 \\
\hline No & 174 & 66 \\
\hline Total & 263 & 100 \\
\hline
\end{tabular}

Fuente: Datos propios de la investigación

Tabla 7: Complicaciones del uso de las plantas medicinales como medicina tradicional en el inicio de parto y culminación del parto en parturientas atendidas en el Hospital II EsSalud Tarapoto 2018

\begin{tabular}{lcc}
\hline \multicolumn{1}{c}{ Inicio de parto } & $\mathbf{N}^{\circ}$ & $\mathbf{\%}$ \\
\hline Espontaneo & 131 & 19 \\
Inducido & 132 & 81 \\
\multicolumn{1}{c}{ Total } & $\mathbf{2 6 3}$ & $\mathbf{1 0 0}$ \\
\hline
\end{tabular}

\begin{tabular}{lcc}
\hline $\begin{array}{l}\text { Forma de } \\
\text { culminación }\end{array}$ & $\mathbf{N}^{\circ}$ & \% \\
\hline Parto natural & 144 & 27 \\
Parto abdominal & 119 & 73 \\
\hline \multicolumn{1}{c}{ Total } & $\mathbf{2 6 3}$ & $\mathbf{1 0 0}$ \\
\hline
\end{tabular}

Fuentes: Datos propios de la investigación 
Tabla 8: Relación entre el uso de las plantas medicinales como medicina tradicional y en el inicio de parto y culminación del parto en parturientas atendidas en el Hospital II EsSalud Tarapoto 2018.

\begin{tabular}{|c|c|c|c|c|c|c|c|}
\hline \multirow{2}{*}{ PARTO } & \multicolumn{6}{|c|}{ USO DE PLANTA MEDICINAL } & \multirow{2}{*}{$\begin{array}{c}X^{2} \\
p<0,05\end{array}$} \\
\hline & \multicolumn{2}{|c|}{$\mathbf{S i}$} & \multicolumn{2}{|c|}{ No } & \multicolumn{2}{|c|}{ Total } & \\
\hline Inicio de parto & $\mathbf{N}^{\circ}$ & $\%$ & $\mathbf{N}^{\circ}$ & $\%$ & $\mathbf{N}^{\circ}$ & $\%$ & \multirow{5}{*}{$\begin{array}{c}X^{2}= \\
215,262 \\
p=0,0000\end{array}$} \\
\hline Espontaneo & 241 & $68.9 \%$ & 13 & $3.7 \%$ & 254 & $72.6 \%$ & \\
\hline Inducido & 20 & $5.7 \%$ & 24 & $6.9 \%$ & 44 & $12.6 \%$ & \\
\hline No refiere & 2 & $0.6 \%$ & 50 & $14.3 \%$ & 52 & $14.9 \%$ & \\
\hline Total & 263 & $75.1 \%$ & 87 & $24.9 \%$ & 350 & $100.0 \%$ & \\
\hline
\end{tabular}

\begin{tabular}{lccccccc}
\hline $\begin{array}{c}\text { Forma de } \\
\text { culminación }\end{array}$ & $\mathbf{N}^{\circ}$ & $\boldsymbol{\%}$ & $\mathbf{N}^{\circ}$ & $\boldsymbol{\%}$ & $\mathbf{N}^{\circ}$ & $\boldsymbol{\%}$ & \\
Parto natural & 178 & $50.9 \%$ & 10 & $2.9 \%$ & 188 & $53.7 \%$ & $\mathrm{X}^{2}=$ \\
Parto abdominal & 82 & $23.4 \%$ & 17 & $4.9 \%$ & 99 & $28.3 \%$ & 208,628 \\
No refiere & 3 & $0.9 \%$ & 60 & $17.1 \%$ & 63 & $18.0 \%$ & $\mathrm{p}=0,0000$ \\
\cline { 1 - 5 } Total & $\mathbf{2 6 3}$ & $\mathbf{7 5 . 1 \%}$ & $\mathbf{8 7}$ & $\mathbf{2 4 . 9 \%}$ & $\mathbf{3 5 0}$ & $\mathbf{1 0 0 . 0 \%}$ & \\
\hline
\end{tabular}

Fuentes: Datos propios de la investigación

\subsection{DISCUSIÓN}

La medicina alternativa y complementaria comprende varios métodos de curación que han sido empleados durante siglos, como las plantas medicinales entre otros. A este método se llama fitoterapia que es uno de los más antiguos, pues las plantas han constituido el recurso más utilizado por la humanidad para comer, vestirse y curar sus enfermedades. Sin embargo, a nivel mundial la Organización Mundial de la Salud ha informado más del $80 \%$ de las personas en los países en desarrollo utilizan la medicina tradicional herbolaria para resolver sus problemas de salud. Por lo cual se realizó el presente estudio en la región selva, específicamente en San Martín, en un establecimiento de salud semiprivado como EsSalud, considerando que las condiciones sociales son diferentes que las mujeres que acuden a los servicios públicos, para conocer el uso de plantas medicinales en la mayoría de parturientas que por la condición cultural es alto, las cuales muchas veces consumen estas plantas sin ayuda profesional y sin ningún estudio previo.

Podemos observar en la tabla 1 que el $72 \%$ de las parturientas según lugar de nacimiento son de San Martín, seguido de Cajamarca con 6\% y de Loreto 10\%. Según región natural en su mayoría fueron de la Región Selva con 87.14\%; y según 
procedencia el $68.57 \%$ proceden de zona urbana seguido de zona rural $30.86 \%$. En el segundo resultado podemos observar que el $47.43 \%$ de las parturientas corresponden al grupo etáreo de 30-39 años, de grado de instrucción en su mayoría con 46\% corresponden a educación superior no universitaria y de ocupación el $66.57 \%$ son empleadas seguido de ama de casa $28 \%$.

Resultado que se relaciona con Condori Z, Orellana S. (2018), quienes estudiaron a mujeres mayores de 30 años de edad en un 47\%, un nivel de educación secundario en un $49 \%$ y multíparas en un 66\%. También se relaciona con León C, Tello H. (1995), quienes en su grupo de población tuvieron primigestas y multigestas. Igualmente se relaciona con Kennedy D, Lupattelli A, Koren G, Nordeng H. (2012), quienes encontraron como características en su población de estudio a mujeres que tenían a su primer hijo, no fumadoras, usaban ácido fólico y consumían algo de alcohol durante el embarazo. Además, eran estudiantes y con una educación que no es un diploma de secundaria.

Por lo mencionado las mujeres que usan plantas medicinales básicamente dentro de sus características resalta la educación superior no universitaria y universitaria y la procedencia urbana, así como la ocupación ya que por ser una institución que brinda atención de seguro social está relacionado con población cerrada de mujeres empleadas o ser esposa de un empleado por lo tanto el uso de las plantas medicinales puede deberse básicamente a la cultura por las tradiciones familiares o por influencia básicamente de la familia de ella o del esposo como son la mamá, suegra, tías u otras.

El tercer resultado nos muestra que el $75 \%$ de las parturientas usan plantas medicinales como medicina tradicional y el uso es mayor en la etapa del embarazo con $38.78 \%$ seguido del posparto con $23.19 \%$. Además, la parte más utilizada de las plantas es el tallo con $39.5 \%$ (104) seguido de las hojas con $27.8 \%$. En cuanto al modo de uso predominó usarlo como infusión con $53.6 \%$, seguido de cocido con $32.3 \%$.

Resultados que se relacionan con Macarro D, Miguelez L, Martínez E, Martínez P, Manrique J. (2014), quienes encontraron que en la etapa del embarazo hay mayor consumo de plantas medicinales, sobretodo en el primer trimestre. El mayor porcentaje de gestantes utiliza plantas sin indicación médica. También se relaciona con MacíasPeacok B, Pérez-Jackson L, Suárez-Crespo M, Fong-Domínguez C, Pupo-Perera E. (2009) quienes encontraron que las embarazadas utilizaban plantas principalmente en el 
primer trimestre. Sin embargo, Kennedy D, Lupattelli A, Koren G, Nordeng H. (2012) encontraron que la mayoría de las hierbas medicinales se usaban para las dolencias de salud relacionadas con el embarazo, el resfrío y las náuseas. También, no se relaciona con Perlaza N. (2016) quien encontró que las plantas medicinales más las usaron durante el parto.

Por lo mencionado podemos establecer que la mayoría de embarazadas usa principalmente las plantas medicinales durante la etapa del embarazo y posteriormente para el parto y postparto, sin embargo, podemos resaltar que al no utilizarlas bajo indicación médica y previo estudio del principio activo de las plantas y al identificarse que lo usan desde el primer trimestre ello puede causar problemas teratógenos en el feto durante la etapa de embriogénesis.

En la tabla 4 se observa que las plantas medicinales más usadas por las parturientas fueron la manzanilla, el anís, la malva y el apio con $22 \%, 17 \%, 13 \%$ y $12 \%$ respectivamente, siento diferente el valor total esperado al número de parturientas porque en su mayoría no toman una sola planta medicinal sino combinan de dos a más plantas. El resultado 5 muestra que las plantas medicinales según indicación y uso corresponde efectivamente analgésico $40.30 \%$ (106) y 39\% (102) respectivamente, digestivo $25.86 \%$ (68) y $24 \%$ (63) respectivamente; relajante $15.59 \%$ (41) y $16 \%$ (42); sólo se identificó diferencia en la indicación y acción espasmódico 9.51\% (25) y 13\% (34) seguido de antiinflamatorio $8.75 \%$ (23) y 5\% (13). El resultado 6 evidencia que el uso de plantas medicinales causó complicaciones en el 34\% (89) parturientas atendidas, seguido del 66\% (174) que no presentó complicaciones. Sin embargo, en la tabla 7 podemos observar que las parturientas que usaron plantas medicinales como medicina tradicional tuvieron un inicio de parto inducido con $81 \%$ (132), seguido de espontáneo con $19 \%$ (31). Según forma de culminación del parto el $73 \%$ (119) termino en parto abdominal (cesárea).

Resultados que se relacionan con Macarro D, Miguelez L, Martínez E, Martínez P, Manrique J. (2014), quienes encontraron como plantas más usadas la equinácea que fue eficaz para el resfriado común e infecciones vaginales, el arándano rojo para la infección urinaria, la raíz de jengibre y la ipecacuana tienen acción antiemética, la hierba de San Juan es eficaz en la depresión postparto, la valeriana para combatir el nerviosismo y el aceite de onagra para inducir el parto. También se relaciona con 
Macías-Peacok B, Pérez-Jackson L, Suárez-Crespo M, Fong-Domínguez C, PupoPerera E. (2009), quienes encontraron que las plantas medicinales más consumidas fueron menta japonesa (Mentha arvensis), tilo (Justicia pectoralis), verbena (Stachytarpheta jamaicensis) y anisón (Piper auritum). Igualmente se relaciona con Perlaza N. (2016) quien encontró que las parteras utilizaron plantas medicinales como: Melloco, Contra-hierba, Linaza, Culantro, Lengua de vaca, Manzanilla, Tama-Tama, Mashua, Zanahoria Blanca, Granizo y Canayuyo, con diferentes fines terapéuticos. Asimismo, se relaciona con Kennedy D, Lupattelli A, Koren G, Nordeng H. (2012), quienes encontraron que la mayoría de hierbas medicinales se usaban para las dolencias relacionadas con el embarazo, el resfrío y las náuseas. El jengibre, el arándano rojo, la valeriana y la frambuesa fueron las hierbas más utilizadas en el embarazo. También se relaciona con Lora E, Narváez L. (2010), encontraron el consumo de aguas aromáticas o infusiones se indica que: de 70 mujeres encuestadas el $44 \%$ tomaron agua de paraguay durante el trabajo de parto, el 35\% consumió agua de panela, y el 9\% consumió agua de anís, $6.7 \%$ consumió agua de ruda, $5.5 \%$ agua de higo. Igualmente se relaciona con Fong C, Macías B, (2004) quienes encontraron que las plantas más consumidas fueron la menta, el tilo o carpintero y la verbena. Las gestantes emplearon además sábila, orégano, albahaca, caña santa entre otras. Asimismo, se relaciona Pulido A, Vásquez S, Villamizar G. (2012), quienes encontraron que las gestantes usaron 18 hierbas como el té de anís, llantén, limonaria, ramo bendito, sauco, cáscara de mandarina y agua con canela durante el primer y tercer trimestre de la gestación es el momento en el que más se utilizan las hierbas. El modo de uso más frecuente corresponde a la infusión y la mayoría de las prácticas son aprendidas de la madre de la gestante. La hierba más utilizada fue la manzanilla (36.7\%) surtiendo el efecto esperado en un 60\% de los casos, usada para acelerar el trabajo de parto, el frío y los 16 cólicos. El uso más frecuente de las hierbas fue para acelerar el trabajo de parto.

Por lo indicado podemos establecer que la mayoría de mujeres usa plantas medicinales durante las diferentes etapas de la concepción siendo principalmente la etapa de gestación la más resaltantes seguido del trabajo de parto básicamente por condiciones de cólico o dolor o secreción o contracción o por inducir a la aceleración del parto, sin embargo a pesar que las plantas no son las mismas en la mayoría de estudios por ser países diferentes y al desconocer su composición y principio activo no podemos saber 
su similitud y efectividad sin embargo todas son usadas en su mayoría por los mismos hechos que nos conlleva a ello, pero no significa efectividad positiva porque encontramos que las mujeres manifestaron complicaciones y entre las que más se resaltan están la inducción del trabajo de parto y la cesárea que pude ser por diferentes motivos y al desconocer la dosis apropiada podemos las madres puede causar teratógenia o iatrogenia al bebe, lo que demanda mayores estudios de las plantas medicinales y mayor educación en las madres para su uso.

El resultado ocho nos muestra la relación que existe entre el uso de las plantas medicinales como medicina tradicional y el inicio y culminación del parto en parturientas atendidas en el Hospital II EsSalud - Tarapoto, se aplicó la prueba no paramétrica chi cuadrado con un nivel de confianza al 95\%, obteniéndose que si existe relación entre el uso de planta medicinal y el inicio $\left(X^{2}=215,262 ; p=0,0000\right)$ y forma de culminación del parto $\left(X^{2}=208,628 ; p=0,0000\right)$ por tener un $p$ valor $<0,05$.

Por lo encontrado podemos establecer que os beneficios de las plantas medicinales contribuyen a la reducción del tiempo del periodo de trabajo de parto y por ende es necesario que puedan analizarse los principios activos de cada planta medicinal que se usaron por las parturientas para poder establecer las dosis y condiciones de uso de una forma científica.

\section{CONCLUSIONES}

Las parturientas se caracterizaron por: tener como lugar de nacimiento el departamento de San Martín 72\%, como región natural la Región Selva 87.14\% y procedencia zona urbana $68.57 \%$, corresponder al grupo etario de 30-39 años 47.43\%; de educación superior no universitaria $46 \%$ y de ocupación empleada $66.57 \%$.

La mayoría de parturientas usan plantas medicinales como medicina tradicional $75 \%$, el uso es mayor en el embarazo $38.78 \%$ seguido del posparto $23.19 \%$. Además, la parte más utilizada de la planta es el tallo $39.5 \%$, seguido de las hojas $27.8 \%$, y en cuanto al modo de uso predomino la infusión $53.6 \%$ seguido de cocido $32.3 \%$.

Las parturientas usaron principalmente como plantas medicinales la manzanilla $22 \%$, el anís $17 \%$, la malva $13 \%$ y el apio 12\%, sin embargo, el valor total esperado según número de parturientas no corresponde porque en su mayoría toman de 2 a más plantas. Los beneficios de las plantas medicinales según indicación y uso corresponden como analgésico $40.30 \%$ y $39 \%$ respectivamente, digestivo $25.86 \%$ y $24 \%$ respectivamente; 
relajante $15.59 \%$ y 16\%; sólo se identificó diferencia en la indicación y acción como espasmódico $9.51 \%$ y $13 \%$ seguido de antiinflamatorio $8.75 \%$ y $5 \%$. Las complicaciones fueron $34 \%$, de las parturientas tuvieron un inicio de parto inducido $81 \%$ y según forma de culminación del parto el $73 \%$ termino en parto abdominal (cesárea).

La mayoría de parturientas usan las plantas medicinales sin indicación y existe relación significativa entre uso de las plantas medicinales como medicina tradicional y el inicio y culminación del parto en parturientas atendidas en el Hospital II EsSalud - Tarapoto, obteniéndose como uso de planta medicinal y el inicio $\left(X^{2}=215,262 ; p=0,0000\right)$ y forma de culminación del parto $\left(\mathrm{X}^{2}=208,628 ; \mathrm{p}=0,0000\right)$ por tener un $\mathrm{p}$ valor $<0,05$.

\section{REFERENCIAS}

García A, Morón F, Larrea C. Plantas medicinales en revistas científicas de Cuba colonial y neocolonial. Scielo. 2010. Octubre-Diciembre.; 15.(4.).

Amaya M, Contreras E. Manual de plantas medicinales para mujeres durante el embarazo, parto y el postparto. [Online]. Gunea Ecuatorial; 2012 [cited 2016 Julio 08. Available from: https://haurdun.files.wordpress.com/2008/11/manual-deplantasmedicinales-para-mujeres-durante-el-embarazo.pdf.

Castro I. Actualidad de la medicina Tradicional Herbolaria. Revistas médicas cubanas. 2006. Abril-Junio.; 11.(2.): p. 8-10

Puerto M, Casas L, Cañete R. Usos más frecuentes de Arnica montana. Revista Cubana de plantas medicinales Scielo. 2013. Abril-Junio.; 18.(2.): p. 4-7.

Macarro D, Miguelez L, Martínez E, Martínez P, Manrique J. Fitoterapia en embarazo y lactancia: ¿beneficio o riesgo? Revista científica de enfermería. ISNN-1989-6409. $\mathrm{N}^{\circ} 9$ Noviembre 2019. España. 2014. Consulta 31.03.19 https://rua.ua.es/dspace/bitstream/10045/45018/1/RECIEN_09_06.pdf

Macías-Peacok B, Pérez-Jackson L, Suárez-Crespo M, Fong-Domínguez C, PupoPerera E. Consumo de plantas medicinales por mujeres embarazadas. Historia y Antropología Médica. Centro de Toxicología y Biomedicina. Rev. Med. Inst. Mex. Seguro Soc. 2009; 47 (3): 331-334. Santiago de Cuba. 2009. Consulta 31.03.19 http://www.medigraphic.com/pdfs/imss/im-2009/im093q.pdf.

Perlaza N. Utilización de plantas medicinales durante el parto tradicional en la comunidad de Oyacachi, Napo 2016. Universidad Técnica del Norte. Facultad 
Ciencias de la Salud. Carrera de Enfermería. Tesis previa la obtención del título de licenciatura en enfermería. Ibarra Ecuador. 2016 Consulta 27.06.18 http://repositorio.utn.edu.ec/bitstream/123456789/5368/1/06\%20ENF\%20748\%20 TESIS\%20DE\%20GRADO.pdf

Kennedy D, Lupattelli A, Koren G, Nordeng H. Uso de hierbas medicinales en el embarazo: resultados de un estudio multinacional. BMC medicina complementaria y alternativa. 13: 355. doi: 10.1186 / 1472-6882-13-355. Europa, América del Norte, América del Sur y Australia. 2012. Consulta 27.06.18 https://www.ncbi.nlm.nih.gov/pmc/articles/PMC4029224/

Lora E, Narváez L. "Creencias y costumbres que poseen las mujeres de la comunidad del chamizo sobre el embarazo parto y puerperio desde enero a junio del 2010". Universidad Técnica del Norte. Facultad de Ciencias de la Salud. Escuela de enfermería. Trabajo de Investigación previo a la obtención del título de Licenciados de Enfermería. Ibarra- Ecuador 2010. Consulta 21.09.17 5:27 am. http://docplayer.es/36952555-Universidad-tecnica-del-norte-facultad-ciencias-dela-salud-escuela-de-enfermeria.html

Fong C, Macías B. Utilización de la fitoterapia en gestantes que acuden al policlínico docente comunitario “Josue País García. Ilustrados. Artículo Científico. Cuba. 2004. Consulta 21.09.17 6:13 am. http://www.ilustrados.com/tema/8467/Utilizacion-fitoterapia-gestantes-areasalud.html

Pulido A, Vázquez S, Villamizar G. Uso de hierbas medicinales en mujeres gestantes y en lactancia en un hospital universitario de Bogotá (Colombia), vol.21, n.4, pp. 199-203. ISSN 1132-1296. 2012. Recuperado el 18-01-2015. Disponible en: http://scielo.isciii.es/scielo.php?pid=S1132-

$12962012000300005 \&$ script=sci_abstract $\&$ tlng=e.

Antamba B. Utilización de las plantas medicinales en el postparto, Cantón el Chaco, Provincia del Napo, período diciembre 2015 - mayo 2016. Universidad Técnica del Norte. Facultad Ciencias de la Salud. Carrera de Enfermería. Tesis previa a la obtención del título de licenciatura en enfermería. Ibarra Ecuador. 2015. Consulta 21.09.17n am. 
http://repositorio.utn.edu.ec/bitstream/123456789/5367/1/06\%20ENF\%20747\%20 TESIS\%20DE\%20GRADO.pdf

Yampis J. Plantas medicinales utilizadas durante el proceso de parto y puerperio en madres de la Comunidad Nativa Awajun de Achoaga, Amazonas 2015. Universidad Nacional "Toribio Rodríguez de Mendoza de Amazonas". Facultad de Ciencias de la Salud. Escuela Profesional de Enfermería. Tesis para optar el título profesional de Licenciado en Enfermería. Amazonaserú 2015. Consulta 17.04.18 $7: 29$

am.

http://repositorio.untrm.edu.pe/bitstream/handle/UNTRM/834/Plantas\%20medicin ales $\% 20$ utilizadas $\% 20$ durante $\% 20 \mathrm{el} \% 20$ proceso $\% 20 \mathrm{de} \% 20$ parto $\% 20 \mathrm{y} \% 20$ puerper io $\% 20$ en $\% 20$ madres $\% 20 \mathrm{de} \% 201 \mathrm{a} \% 20$ comunidad $\% 20$ nativa $\% 20$ Awajun $\% 20 \mathrm{de} \% 2$ 0Achoaga,\%20Amazonas\%202015.pdf?sequence $=1$

Solis P, Tapia L. Prácticas relacionadas con el uso de plantas medicinales en el trabajo de parto y puerperio Puesto de Salud Miramar Región La Libertad abril 2015. Universidad Privada Antenor Orrego. Facultad de Ciencias de la Salud. Escuela Profesional de Obstetricia. Tesis para optar el Título Profesional de Obstetra. Trujillo Perú. 2015. Consulta 17.04.18 7:38 am. http://repositorio.upao.edu.pe/bitstream/upaorep/1121/1/SOLIS_PAOLA_PLANT AS_MEDICINALES_PARTO.pdf

Condori Z, Orellana S. Influencia del uso de infusiones de plantas medicinales en el trabajo de parto en pacientes atendidas en el Centro de Salud de Chilca, en el periodo de abril - septiembre del 2017. Universidad Privada de Huancayo Franklin Roosevelt. Facultad de Ciencias de la Salud. Escuela Académica Profesional de Obstetricia. Tesis de pregrado. Huancayo Perú. 2018. Consulta 31.03.19 http://webcache.googleusercontent.com/search?q=cache:mcc5DOjt0cJ:repositorio.uroosevelt.edu.pe/xmlui/handle/ROOSEVELT/98+\&cd=2\& $\mathrm{hl}=\mathrm{es} \& \mathrm{ct}=\mathrm{clnk} \& \mathrm{gl}=\mathrm{pe}$

León C, Tello H. Uso de la infusión de hoja de algodón (Gessypium peruvianum) y su relación con la duración del trabajo del parto en gestantes atendidas en el Hospital Regional de Loreto. Universidad Nacional de San Martín Tarapoto. Facultad de Obstetricia. Tesis para optar el título professional de Obstetriz. San Martín - Perú. 
1995.

Consulta

17.04.18

$9: 43$

pm.

http://repositorio.unsm.edu.pe/handle/UNSM/2281

Medina I, Mayca J. Creencias y costumbres relacionadas con el embarazo, parto y puerperio en comunidades nativas awajun y wampis. Rev Peru Med Exp Salud Publica 23(1). Trabajo original. Lima Perú. 2006. Consulta 21.09.17. 4:40 am http://www.scielo.org.pe/pdf/rins/v23n1/a04v23n1.pdf

Cabieses F. Apuntes de Medicina Tradicional: la racionalización de lo irracional. Tomo I. Lima: DISELPESA; 1993.

Ministerio de Salud / Proyecto 2000. Salvarse con bien: El parto de la vida en los Andes y la Amazonía del Perú. Técnicas tradicionales de atención del parto y del recién nacido. Lima: MINSA; 1999.

Dibbits I, de Boer M. Encuentros con la propia historia. Interculturalidad y trato humano desde las perspectivas de un equipo de salud. La Paz: TAHIPAMU; 2002.

Regan J. Mito y rito: Una comparación entre algunas imágenes Mochicas y Jíbaras. Investig Soc 1999; 3(3): 27-46.

Vargas R, Naccarato P. Allá las antiguas abuelas eran parteras. Etnografía de las parteras empíricas. Lima: Centro de la Mujer Peruana Flora Tristán; 1995

Frisancho P. ¿Qué es la Medicina Folklorica? Lima: Ediciones Hampicamayoc S.A.; 1999.

Llumiquinga D. Conocimientos y prácticas ancestrales del parto tradicional en las mujeres de la cultura Saraguro año 2011. [Online][Doctorado] Ortiz E, editor. Loja; 2011.

Almeida I, Arrobo N, Ojeda L. Autonomía indígena: frente al estado nación y la globalización neoliberal. Primera ed. Guerrero J, editor. Quito: ABYA-YALA; 2005

Arrobo N. Las culturas indígenas y sus saberes ancestrales. Llacta. 2005 Enero; (1).

Centro Nacional de Información sobre la Salud de la Mujer (julio de 2007). Parto. Citado por National Institutes of Health. Último acceso 20 de febrero de 2008.

Liljestrand J. Episiotomía en el parto vaginal. Comentario de La Biblioteca de Salud Reproductiva de la OMS. Última revisión: octubre 2013. Ginebra: Organización Mundial de la Salud. 
The Nemours Foundation - kidsHealth.org (junio de 2006). Parto Natural.. Último acceso 20 de febrero de 2008 .

Adrià Palau Miguel (Victoria Amat): Parto y etapas del parto. Consulta 24.07.19 https://www.google.com/search?q=Adri\%C3\%A0+Palau+Miguel+(Victoria+Amat \%3A+Pelvis+femenina.+22\%2F10\%2F14\&rlz=1C1CHBD_esPE805PE805\&oq= Adri\%C3\%A0+Palau+Miguel+(Victoria+Amat)\%3A+Pelvis+femenina.+22\%2F10 \%2F14\&aqs=chrome..69i57.1230j0j7\&sourceid=chrome \&ie=UTF-8

Salud 180. Estilos de vida saludable. Clasificación del Trabajo de Parto. México y $\begin{array}{lllll}\text { América } & \text { Latina. } & \text { Consulta } & \text { 21.09.17 } & \end{array}$ http://www.salud180.com/maternidad-e-infancia/clasificacion-del-trabajo-de-parto Galvez I, Lobos G, Peralta J. Plantas medicinales, principios básicos de fitoterapia. Primera ed. Quito. 2014. Consulta 21.09.17 3:33 pm http://www.saludterapia.com/glosario/d/33-fitoterapia.html

López M. Manual de plantas medicinales para ginea Ecuatorial. Primera ed. Canellas Garcia C, editor. Fundación de religiosos para la salud. 2012. Consulta 21.09.17 $3: 36$

$\mathrm{pm}$

http://www.academia.edu/6947967/MANUAL_DE_PLANTAS_MEDICINALES_ PARA_GUINEA_ECUATORIAL

López T. Plantas medicinales en la medicina tradicional china. Fitoterapia. 2008 Febrero. 22(2). Consulta 21.09.17 3:40 pm. http://www.elsevier.es/es-revistaoffarm-4-articulo-las-plantas-medicinales-medicina-tradicional-13043202

Zhang X. Situación reglamentaria de los medicamentos herbarios. Primera ed. Steinhoff B. Editor. 2005. Consulta 21.09.17 3:45 pm. http://apps.who.int/medicinedocs/es/d/Jwhozip58s/

Bagozzi D. Organizacion Mundial de la Salud. [Online].; 2004 [cited 2015 Octubre 05.

Available from: http://www.who.int/mediacentre/news/releases/2004/pr44/es/.

Goulart de Faria P, Ayres A, Titonelli N. O diálogo com gestantes sobre plantas medicinais: contribuicoes para os cuidados básicos de saúde. Scielo. 2007; 26(2).

López M. Manual De Plantas Medicinales Para Guinea Ecuatorial, Fundación de religiosos para la Salud, 1 era Edición España, FRS. 2012.

Forster D, Denning A, Wills G, Bolger M, McCarthy E. Herbal medicine use during pregnancy in a group of Australian women. BMC Pregnancy and Childbirth. 2006. 
pm.

https://www.ncbi.nlm.nih.gov/pmc/articles/PMC1544352/

Klein S, Miller S, Thomson F. Un libro para parteras; Atención del embarazo, el parto y la salud de la mujer Wallis S, editor. California: Hesperian; 2013.

Cameroni G. Ficha Técnica: Manzanilla (matricaria recutita) cadena hierbas aromáticas y especias Rubio M, editor. Santiago de Chile

Dueñas R. Manzanilla. Laboratorio de remedios herbolarios fabricación de extractos fluidos y secos. 2014 Enero; 1.

Aguirre Z. Especies forestales bosques secos Ecuador. Primera ed. Jadan O, editor. Quito: Abya - Yala; 2012.

Fonnegra G, Jiménez S. Plantas medicinales aprobadas en Colombia. Segunda ed. Cárdenas Mesa ÉA, editor. Antioquia: Universidad de Antioquia; 2007

Martí H, Corbino G, Chludil H. La batata: el redescubrimiento de un cultivo. Batatas. 2011 Febrero; 21(121).

Bye L, Ramírez R, Pereda M. El camote. CONABIO - Biodiversitas. 2008; Enero (81).

Hall Ramìrez V, Rocha Palma M, Rodríaguez Vega E. Plantas medicinales. CIMED Centro de información de medicamentos. 2006 Mayo; II.

Gutiérrez Sullca JR. Eficacia de cicatrización con el aceite esencial cinnamomum zeylanicum (canela) versus el apósito convencional (COEPAK) en ratas albinas.

Primera ed. Chuna Espinoza J, editor. Lima: Universidad nacional Federico Villarreal; 2011.

Bonilla L, García R. La medicina ancestral como recurso potencial para el desarrollo del turismo comunitario en la zona andina, del Cantón Cotacachi, Provincia de Imbabura [Online] (Licenciatura) Chorlango G, editor. Cotacachi; 2009.

Trujillo Santillán NS. Utilización de eucalipto (Eucalyptus citriodora) como promotor del crecimiento en dietas para pollos de engorde [Online] (Doctorado) Guerrero López JR, editor. Ambato; 2015.

Andrade Cetto A. Ethnobotanical study of the medicinal plants from Tlanchinol, Hidalgo, México. Primera ed. México DF: Journal of Etnopharmacology; 2008.

León Hernández J, Valero H, Gil Otaiza R. 23 Especies vegetales medicinales de uso frecuente en la población de Tabay. Facultad de Farmacia. 2005; 44. 
Sostenible M. Producción, aprobechamiento y uso de especies aromaticas y medicinales - descripción y uso de 12 especies aromáticas y medicinales Lucero LF, editor. La Paz: Aguila Editores s.r.1.; 2007.

Avila Sosa R, Navarro Cruz AR, Vera López O, Dávila Márquez RM, Melgoza Palma N, Meza Pluma R. Romero (Rosmarinus offinalis L.): una revisión de sus usos no culinarios. Ciencia y mar. 2011; XV(43).

Alarcón J. Plantas aromáticas y medicinales - Enfermedades de importancia y sus usos terapéuticos Vásquez González CE, editor. Bogota: Produmedios; 2011.

Charco J. Evaluación de la actividad cicatrizante de un gel elaborado a base de los extractos de guarango (caesalpinia spinosa), nogal (juglans regia) y tomillo (thymus vulgaris) en ratones (mus musculus) [Online] (Licenciatura) Toapanta G, editor. Riobamba; 2015

Linares N. Plantas medicinales - cuaderno de trabajo. Primera ed. Madrid; 2013.

Martínez N. Plantas medicinales - parte 2. SLAN. 2005 noviembre;(4).

Narváez P. Taller plantas medicinales Martínez P, editor. Tamarindo: Fundación puente; 2006. 